\title{
Incremento y rendimiento maderable de Pinus montezumae Lamb., en San Juan Tetla, Puebla
}

\author{
E. Marcelo Zepeda Bautista ${ }^{1}$ \\ Miguel Acosta Mireles ${ }^{2}$
}

\begin{abstract}
RESUMEN
Se presentan resultados de un análisis dasométrico de datos de parcelas permanentes de observación silvícola, establecidas en el Campo Experimental Forestal San Juan Tetla, municipio de Chiautzingo, Puebla, a principios de la década de los 70's, consistentes en ecuaciones dasométricas que permiten conocer el comportamiento promedio, a través del tiempo, del incremento $y$ rendimiento maderable y la sobrevivencia probable, de las poblaciones arboladas representadas en los datos.
\end{abstract}

Tales ecuaciones permiten conocer inicialmente los incrementos y rendimientos maderables netos de los rodales representados en la muestra, con la consiguiente ventaja para el manejo silvícola operativo de las poblaciones de Pinus montezumae Lamb., Chiautzingo, Puebla, México.

\section{PALABRAS CLAVE:}

Modelo compatible, crecimiento, rendimiento, madera, ecuación dasométrica, Pinus, Pinus montezumae.

\begin{abstract}
A dasometric analysis using data from permanent plots established during the early 70 's at San Juan Tetla Forest Experimental Camp, Puebla State, was made. The results included a set of dasometric equations which allow us to estimate the average behavior through time, of growth, yield and probable survival of the stands represented in the sample.

Such equations initially allow us to know the net growth and yield wood of those stands, with the obvious advantage for the operative silvicultural management of stands of Pinus montezumae Lamb., Chiautzingo, Puebla, México.
\end{abstract}

KEY WORDS:

Compatible, model, growth, yield, wood, dasometric equation, Pinus, Pinus montezumae.

1 Universidad Autónoma Chapingo. Div. Ciencias Forestales. Apdo. Postal 37. Chapingo, Edo. de México. 56230 México. c.e.: zepedabm@.taurus1.chapingo.mx

2 Colegio de Posgraduados. Programa Forestal. Km. 35.5 Carr. Mèxico-Texcococ. Montecillo, Edo de México. 56230 México. c.e.: acostamm@colpos.colpos.mx

Recibido el 27 de marzo de 1999. Aceptado el 20 de octubre de 1999 


\section{INTRODUCCIÓN}

Los rendimientos maderables de algunas coníferas mexicanas son notables en ciertas condiciones biofísicas del territorio nacional y para algunas existen datos provenientes de parcelas permanentes con las que es posible construir ecuaciones de pronóstico iniciales, que permitan mejorar las estimaciones del rendimiento maderable para preparar, más adelante, calendarios de cosecha basados en criterios de rentabilidad financiera, más allá de estimaciones de volúmenes de corta calculados sobre la base del "criterio profesional"; también para estimar rendimientos probables de plantaciones comerciales, susceptibles de ser establecidas en tales localidades.

Adicionalmente, la necesidad de superar algunos métodos empíricos de aprovechamiento maderable, o al menos mejorar las estimaciones que sirven de base para fincar sus decisiones de aprovechamiento, es impostergable.

Por otra parte, el requerimiento de manejar las poblaciones arboladas sobre una base tal que permita al administrador del bosque controlar algunos de los factores que gobiernan las respuestas del incremento y rendimiento maderable de tales rodales, para hacer más eficiente el proceso productivo forestal, mueve a mejorar necesariamente las estimaciones tradicionales de rendimiento maderable, que permitan, a su vez, avanzar en los sistemas de planeación utilizados para el manejo forestal de tipo perdurable.

Los resultados de este trabajo proporcionan información que permite la comparación de las tasas de incremento y rendimiento maderable de Pinus montezumae Lamb, del Plan de Marines, Puebla, con las de otras especies y regiones forestales importantes del país y contribuir a la explicación de las relaciones entre rendimiento maderable y densidad, para la calidad de sitio en que crecen los rodales representados en la muestra.

\section{OBJETIVOS}

a. Traducir ciertos atributos dasométricos de la condición silvícola presente en los datos muestra, en coeficientes de regresión muestral de ecuaciones con un cierto significado biológico, que permita comprender las propiedades biológicas de la función de producción lograda.

b. Generar un sistema de ecuaciones que permita estimar los rendimientos maderables a través del tiempo y realizar un análisis de los efectos de la densidad neta de los rodales y de la calidad de sitio, sobre el incremento y el rendimiento maderable de Pinus montezumae Lamb., del Plan de Marines, Chiautzingo, Puebla.

\section{METODOLOGÍA}

Características del área de estudio (Acosta, 1991)(Fig. 1). El Plan de Marines se ubica en la envolvente del Campo Experimental Forestal San Juan Tetla (CEFSJT), Chiautzingo, en el estado de Puebla, dependiente del Instituto Nacional de Investigaciones Forestales, Agropecuarias y Pesqueras (INIFAP), situado en la cordillera Neovolcánica, en el declive oriental del Iztaccíhuatl, $25 \mathrm{~km}$ al suroeste de San Martín Texmelucan. Las 1,568 ha del CEFSJT limitan al norte con el ejido San Felipe Teotlalzingo y la exhacienda Vaquero; al sur con las exhaciendas Buena Vista, San Luis Coyotzingo, Noeopopualco y San Antonio Tlatenco; al este con la ex-hacienda la Unión, San Felipe Teotlalzingo (ampliación), ejido San Juan Tetla, San Agustín Atzompa y San Antonio Tlatenco (ampliación); y al oeste con los bosques del Parque Nacional Izta-Popo. 


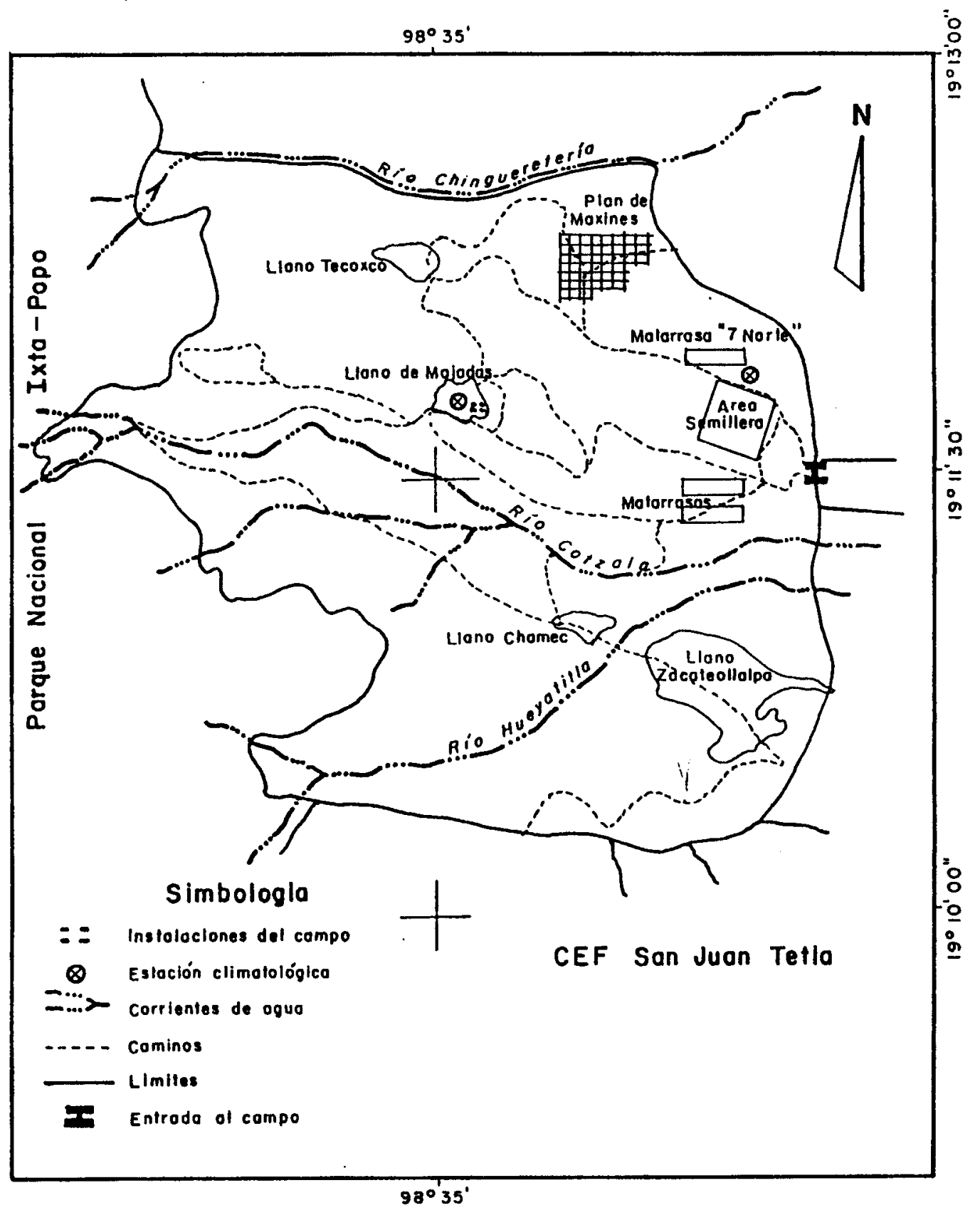

Figura 1. Ubicación del área de estudio en el Campo Experimenta Forestal, San Juan Tetla, Puebla (Acosta, 1991). 
En el área predomina la exposición este, con una pendiente media de 30 a $40 \%$. Existen barrancas, algunas de más de $100 \mathrm{~m}$ de profundidad.

Los suelos se clasifican como de ando o húmicos de alófano. Tienen perfil $A(B) C$ y $A C$ profundo, con horizonte $A$ de color negro o café oscuro, con alto contenido de materia orgánica y abundante presencia de raicillas y macroorganismos. El horizonte $B$ se distingue del A por el color y porque tiene estructura subangular media, fina. El horizonte $\mathrm{C}$, claramente diferenciado, es café amarillento, sin estructura, pero la penetración de las raíces en algunos casos es superior a $1.50 \mathrm{~m}$. La textura es migajón-arenosa. Los valores de $\mathrm{pH}$ son cercanos a la neutralidad dentro del bosque de Pinus; los bosques de Abies son más ácidos.

Se presentan heladas en un promedio de 100 días al año, la temperatura media anual es de $9.2^{\circ} \mathrm{C}$, con mínima de $5^{\circ} \mathrm{C}$ bajo cero, de noviembre a febrero. La precipitación se concentra de mayo a octubre, con $849.6 \mathrm{~mm}$, que significa el 84.2 $\%$ del total, y de noviembre a abril 159.4 $\mathrm{mm}$. Las lluvias máximas mensuales son en junio, con $215.7 \mathrm{~mm}$ y las mínimas en diciembre, con $11.9 \mathrm{~mm}$.

En el CEFSJT existen cuatro asociaciones vegetales arbóreas principales:

a) La asociación de $P$. montezumae, donde se ubica el área experimental motivo de este trabajo, ocupa el segundo lugar en extensión y se encuentra en las áreas de menor pendiente, con suelos más profundos y con cantidad mínima de rocas aflorantes. Sus límites altitudinales son de $2,700 \mathrm{~m}$, donde se mezcla con $P$. teocote, y de $3,450 \mathrm{~m}$, donde predomina el $P$. hartwegii.

b) La asociación de $P$. ayacahuite var. veitchii es la menos definida y de menor extensión y se localiza donde la humedad ambiental es mayor. Los límites altitudinales son de 2,720 m, donde está acompañada con $P$. leiophylla y $P$. teocote y de $3,350 \mathrm{~m}$, donde $P$. montezumae y $A$. religiosa son abundantes.

c) La asociación de $A$. religiosa se encuentra en barrancas, cañadas y laderas con exposición norte, en donde la humedad es la más elevada. Sus límites altitudinales son 2,800 m y $3,600 \mathrm{~m}$.

d) La asociación de $P$. hartwegii es la de mayor extensión y se localiza en la parte más alta del CEFSJT, con mayor pendiente, suelos poco profundos y gran cantidad de rocas aflorantes. Sus limites altitudinales son de 3,400 a $4,000 \mathrm{~m}$. Además, existen otras asociaciones vegetales en el área, como los zacatonales, principalmente en los llanos de mayor altitud, en los cuales las especies predominantes son: Festuca amplissima, $F$. tolucensis, Poa conglomerata, Caolomagrostis tolucensis y otras; matorrales secundarios que se encuentran en la parte más perturbada del bosque y en algunos lugares en donde un incendio destruyó la vegetación primitiva, con especies como Ceanothus coeruleus, Satureja macrostema, Symphoricarpus microphyllus, Eupatrorium glabratum, E. pazcuarense, Senecio spp. y otras.

Características de las masas arboladas del Plan de Marines y variación de la muestra. La base de datos empleada proviene de 64 parcelas experimentales de $2,500 \mathrm{~m}^{2}(50 \times 50 \mathrm{~m})$, establecidas a mediados de 1974 en el llamado Plan de Marines, dentro del CEFSJT, Puebla, un rodal natural maduro de Pinus montezumae Lamb., coetáneo, homogéneo, de alta densidad y poco perturbado, de más de 16 ha de extensión, aunque el área de influencia de las parcelas es de poco más de 800 ha, de poblaciones arbóreas de 
características similares a las de la mesa de interés, ubicadas a su alrededor. En la Tabla 1 se muestra el rango de variación de la muestra disponible para la obtención de las ecuaciones que sirven de base a este trabajo.

\section{Lógica de construcción del sistema de ecuaciones para estimar rendimientos} maderables. Se adoptó la metodología presentada por Zepeda y Domínguez (1998), basada en la propuesta de Clutter (1963), omitiendo al indicador de calidad de sitio de la expresión base, pues el modelo de incremento en área basal presentó inconsistencias en esa variable, ya que el coeficiente de regresión muestral asociado no presentó la significancia estadística mínima aceptable.
Se procedió a eliminar al índice de sitio para las condiciones actuales de la muestra, sin que por ello la expresión resultante pierda compatibilidad analítica y demás propiedades del enfoque metodológico adoptado; además de que tal variable se incorporó finalmente al modelo para estimar el volumen total (modelo 3).

Partiendo de la relación base:

$$
\frac{d A B}{d E}=-\frac{A B \ln (A B)}{E}+c_{1} \frac{A B}{E}
$$

procediendo como en Clutter (1963), se tiene que:

$$
\ln \left(A B_{2}\right)=\frac{E_{1}}{E_{2}} \ln \left(A B_{1}\right)+c_{1}\left(1-\frac{E_{1}}{E_{2}}\right)
$$

Tabla 1. Rango de variación de algunas variables dasométricas de las 64 parcelas permanentes de Pinus montezumae Lamb., establecida en el Plan de Marines,

Chiautzingo, Puebla

\begin{tabular}{|c|r|r|r|r||}
\hline \hline VARIABLE & \multicolumn{1}{|c|}{ MÍNIMO } & \multicolumn{1}{|c|}{ MÁXIMO } & PROMEDIO & VARIANZA \\
\hline E1 & 48.800 & 87.100 & 70.470 & 68.734 \\
\hline E2 & 63.800 & 102.100 & 85.470 & 68.734 \\
\hline N1 & 108.000 & 260.000 & 182.062 & 865.519 \\
\hline N2 & 108.000 & 236.000 & 168.812 & 732.027 \\
\hline HD1 & 25.900 & 35.000 & 31.803 & 3.619 \\
\hline HD2 & 29.700 & 38.100 & 35.710 & 3.105 \\
\hline IS & 21.700 & 34.500 & 28.476 & 5.575 \\
\hline AB1 & 17.510 & 33.778 & 25.118 & 17.598 \\
\hline AB2 & 19.870 & 37.913 & 28.726 & 21.212 \\
\hline VOL1 & 214.078 & 478.147 & 341.935 & 4712.314 \\
\hline VOL2 & 292.850 & 648.186 & 469.602 & 7275.800 \\
\hline \hline
\end{tabular}

E1, E2: edad promedio inicial y final 1 (años) N1, N2: Núm. de árboles por hectárea en los tiempos uno y dos

HD1, HD2: Altura dominante inicial y final (m) IS: Indice de sitio (m)
VOL1, VOL2: Existencias reales iniciales y finales $\left(\mathrm{m}^{3} \mathrm{sc} / \mathrm{ha}\right)$ sc: Sin corteza.

Fuente: Acosta, 1991.

$A B 1, A B 2$ : Area basal inicial y final $\left(\mathrm{m}^{2} / \mathrm{ha}\right)$ 
Una vez estimado " $c_{1}$ " de (2), (3) se ajustó al conjunto de datos disponible:

$$
\ln (V)=B_{0}+B_{1} \mid S+\frac{B_{2}}{E}+B_{3} \ln (A B)
$$

Los valores de 'IS' fueron obtenidos a partir de la ecuación de diferencia algebraica generada por Acosta (1991), para estimar crecimientos en altura dominante para Pinus montezumae Lamb., en el Campo Experimental Forestal San Juan Tetla, Puebla, la cual adoptó las formas:

$$
\begin{aligned}
& I S=42.617725\left(1-e^{-0.031214^{*} E}\right)^{a} \\
& H d=42.617725\left(1-e^{-0.031214^{*} E}\right)^{b}
\end{aligned}
$$

Donde:

$$
\begin{aligned}
a & =\frac{\ln \frac{H d}{42.617725}}{\ln \left(1-e-0.31214^{\star} E_{1}\right)} \\
b & =\frac{\ln \frac{I S}{42.617725}}{\ln \left(1-e^{\left.-0.031214^{*} E_{b}\right)}\right.} \\
H d & =\text { altura dominante (m), } \\
E^{-} & =\text {edadice de sitio (m), (años), y } \\
E_{b} & =\text { edad base }(60 \text { años). }
\end{aligned}
$$

Derivando (3) con respecto al tiempo, se tiene que:

$$
\frac{d V}{d E}=V\left\{-\frac{B_{2}}{E^{2}}+\frac{B_{3}\left(\frac{d A B}{d E}\right)}{A B}\right\}
$$

De las expresiones anteriores, se tiene finalmente que:

$$
\ln \left(V_{2}\right)=b_{0}+b_{1} I S+\frac{b_{2}}{E_{2}}+b_{3} \frac{E_{1}}{E_{2}} \ln \left(A B_{1}\right)+b_{4}\left(1-\frac{E_{1}}{E_{2}}\right)
$$

Con lo que es posible estimar, con el conjunto de ecuaciones, además del incremento instantáneo en área basal y volumen, el área basal y el volumen futuros, a partir del área basal y edad actuales.

Conviene hacer notar que únicamente se ajustaron a los datos (2) y (3), y que (1), (4) y (5) se obtuvieron algebraicamente, lo que ocasiona ciertos problemas de orden estadístico. Sin embargo, fue la única manera de ajustar satisfactoriamente las expresiones de interés al conjunto actual de datos disponibles; situación que deberá mejorarse en el futuro, una vez que se cuente con más remediciones de las parcelas.

Para la ecuación que estima la sobrevivencia promedio, se supuso que la tasa de mortalidad ( $\mathrm{dNo} / \mathrm{dE})$, está relacionada con la edad promedio del rodal (E), para el índice de sitio promedio, de la siguiente manera (Clutter et al., 1983):

$$
\frac{\left(\frac{d N_{0}}{d E}\right)}{N_{0}}=B_{0}
$$


Expresión que integrada, con la condición de que $\mathrm{N}_{2}=\mathrm{N}_{1}$, cuando $\mathrm{E}_{2}=\mathrm{E}_{1}$, corresponde a la siguiente ecuación de diferencia algebraica (Clutter et al., 1983):

$$
N_{2}=N_{1} * e^{B_{0}\left(\ln \left(E_{2}\right)-\ln \left(E_{1}\right)\right)}
$$

Donde:

$$
\begin{aligned}
\mathrm{dAB} / \mathrm{dE}= & \text { tasa instantánea, corriente o } \\
& \text { anual, del incremento en área } \\
& \text { basal } \\
\mathrm{AB}= & \text { área basal } \\
\mathrm{E}= & \text { edad del rodal } \\
\mathrm{V}= & \text { existencias volumétricas del } \\
& \text { rodal } \\
\mathrm{IS}= & \text { índice de sitio } \\
\mathrm{Hd}= & \text { altura dominante }
\end{aligned}
$$

$$
\begin{aligned}
\mathrm{dV} / \mathrm{dE}= & \text { tasa instantánea de incremento } \\
& \text { en volumen } \\
\mathrm{N}= & \text { número de árboles por } \\
& \text { hectárea } \\
\mathrm{In}= & \text { Logaritmo natural del } \\
& \text { argumento. Los subíndices en } \\
& \text { 'AB', 'E', 'V' y ' } \mathrm{N} \text { ', de las } \\
& \text { expresiones (2), (5) y (7), } \\
& \text { indican el inicio y final del } \\
& \text { período de estimación del } \\
& \text { atributo dasométrico de interés. }
\end{aligned}
$$

\section{RESULTADOS Y DISCUSIÓN}

Las ecuaciones obtenidas, usadas para analizar el comportamiento del incremento y rendimientos maderables, corresponden a:

$$
\begin{aligned}
& \ln (V)=2.62698693+0.00942854(I S)+1.03088580 * \ln (A B)-\frac{28.78475238}{E} \\
& r^{2}=0.9927 \\
& \mathrm{CME}=0.05749 \\
& \mathrm{Pr}>\mathrm{F}: 0.0001 \\
& E E b_{0}=0.0552045
\end{aligned}
$$

$$
\ln \left(A B_{2}\right)=\frac{E_{1}}{E_{2}} * \ln \left(A B_{1}\right)+4.616781230\left(1-\frac{E_{1}}{E_{2}}\right)
$$

$$
\begin{aligned}
& r^{2}=0.9956 \\
& \mathrm{CME}=0.19016 \\
& \operatorname{Pr}>\mathrm{F}: 0.000 \\
& E \mathrm{~b}_{0}=0.03853459 \\
& \operatorname{Pr}>|t|: 0.0001 \\
& \ln \left(V_{2}\right)=2.62699+0.00943(I S)-\frac{28.78475}{E_{2}}+1.03089 \frac{E_{1}}{E_{2}} * \ln \left(A B_{1}\right)+4.75939\left(1-\frac{E_{1}}{E_{2}}\right) \\
& \frac{d A B}{d E}=\frac{(A B)}{E[4.616781230-\ln (A B)]} \\
& \frac{d V}{d E}=V\left\{\frac{28.78475}{E^{2}}+\frac{1.03089 \frac{d A B}{d E}}{A B}\right\}
\end{aligned}
$$




$$
N_{2}=N_{1}\left\{e^{-0.3480323^{*}\left[\ln \left(E_{2}\right)-\ln \left(E_{1}\right)\right]}\right\}
$$

Donde:

$$
\begin{aligned}
& \mathrm{d} A \mathrm{~B} / \mathrm{dE}=\text { crecimiento instantáneo en } \\
& \text { área basal }\left(\mathrm{m}^{2} / \mathrm{ha} / \mathrm{año}\right) \\
& A B=\text { área basal }\left(\mathrm{m}^{2} / \mathrm{ha}\right) \\
& \mathrm{E}=\text { edad promedio (años) }
\end{aligned}
$$

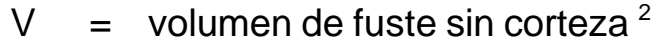

$$
\begin{aligned}
& \left(\mathrm{m}^{3} / \mathrm{ha}\right) \\
& \text { IS = indice de sitio }(\mathrm{m}) \\
& \mathrm{dV} / \mathrm{dE}=\text { crecimiento instantáneo en } \\
& \text { volumen ( } \left.\mathrm{m}^{3} / \mathrm{ha} / \mathrm{año}\right) \\
& \mathrm{N}=\text { número de árboles por } \\
& \text { hectárea; } \\
& \text { In = logaritmo natural del } \\
& \text { argumento. }
\end{aligned}
$$

Los subíndices corresponden a los atributos estimados en los tiempos "1" y "2". En la Tabla 2 se concentran los parámetros e índices de bondad de ajuste de las ecuaciones logradas.
Por el momento, las ecuaciones logradas tienen sus mejores comportamientos en los siguientes rangos: AB: 5 a $45 \mathrm{~m}^{2} /$ ha; EDAD: 10 a 80 años; IS: 20 a $35 \mathrm{~m}$. En el caso particular de la ecuación (13), es preciso hacer notar que tiene comportamientos ilógicos a edades inferiores a los 15 años.

En los cálculos que sirvieron de base para preparar las figuras que se muestran más adelante, el coeficiente de (9) se limitó a $4.45^{3}$, ya que valores de éste iguales a 5 unidades hacen suponer áreas basales de $148.41 \mathrm{~m}^{2} / \mathrm{ha}$, imposibles de lograr en la realidad si se considera, por ejemplo, que las plantaciones Esteafricanas de alto rendimiento exhiben áreas basales máximas de 64,68 y $86 \mathrm{~m}^{2} / \mathrm{ha}$, para Cupressus sp., $P$. patula y $P$. radiata, respectivamente (Alder, 1979). En este caso se asumen

Tabla 2. Concentrado de parámetros e indicadores de bondad de ajuste de las ecuaciones obtenidas

\begin{tabular}{|c|c|c|c|c||}
\hline MODELO No. & $r^{2}$ & CME & Pr $>$ F & PARAMETROS \\
\hline 2 & 0.9927 & 0.190161 & 0.0001 & $c_{1}=4.61678123$ \\
\hline & & & & $\begin{array}{l}b_{0}=2.62698693 \\
b_{1}=0.00942854 \\
b_{2}=1.03088580 \\
b_{3}=28.78475238\end{array}$ \\
\hline
\end{tabular}

Tomado de Acosta (1991).

2 Es claro que no es el mejor tipo de volumen. Sin embargo, fue el disponible por la ecuación de volúmenes empleada por Acosta (1991).

3 La restricción a 4.45 no implica alteración alguna en la estructura o arquitectura del modelo; únicamente es un valor asintótico superior al que se restringe el cálculo. Si se desea, se puede emplear el valor "4.616781230", sin embargo, se decidió restringir las estimaciones a niveles que se consideraron razonables, hasta no contar con mayores evidencias sobre áreas basales de saturación excepcionales. 
áreas basales límite extremas de 85.62 $\mathrm{m}^{2} / \mathrm{ha}^{4}$, que se consideraron razonables ${ }^{5}$.

El coeficiente de (8), para la variable "IS", muestra poca contribución de esa variable a la explicación de la variación total del volumen por hectárea, en la muestra usada en la construcción de las ecuaciones; situación que se atribuye a la homogeneidad en la calidad de sitio del Plan de Marines.

Este mismo coeficiente de (8), asociado al área basal, confirma la relación alométrica existente entre ésta y el volumen por unidad de área, existente en masas homogéneas, y representa el valor de la elasticidad del volumen con respecto al área basal por hectárea (Zepeda y Domínguez, 1998).

Los valores de los coeficientes de la ecuación de sobrevivencia sugieren que la proporción de mortalidad promedio es acelerada en las poblaciones de $P$. montezumae, seguramente por sus rendimientos y velocidades de saturación de los espacios de crecimiento disponibles, también aceleradas, una vez que los árboles superan el estado "cespitoso".

En las figuras 2 a 5 se muestra el comportamiento de algunas variables dasométricas, generado con las ecuaciones anteriores, con la restricción de $c_{1}=4.45$. Por su parte, en las figuras 6 a 9 se presentan los comportamientos espaciales de algunas variables de interés, para el índice de sitio de $25 \mathrm{~m}$ y con la restricción de $\mathrm{c}_{1}=4.45$.

El comportamiento espacial de las diferentes ecuaciones es satisfactorio, lo que evidencia la buena estructura del modelo propuesto, aunque las estimaciones están sujetas a las limitaciones de la muestra. La estimaciones no pueden considerarse pésimas, pero tampoco inmejorables. Los resultados de pronósticos para basar decisiones silvícolas, deben usarse con las reservas del caso.

Como ejemplo de esto último, nótese que el incremento instantáneo en área basal, para el área basal límite seleccionada (85.62 $\mathrm{m}^{2} / \mathrm{ha}$ ), culmina en un nivel de densidad de alrededor de los $30 \mathrm{~m}^{2} / \mathrm{ha}$, aunque en edad no lo hace, pues la ecuación (11) no presenta un cambio en su concavidad, conforme la edad tiende a cero (Fig. 2), lo cual deberá mejorarse en futuras versiones de este sistema de ecuaciones.

El uso práctico de las ecuaciones aquí presentadas debe limitarse, por ahora, a las condiciones silvícolas en el rango de variación de las variables dasométricas reportadas en la Tabla 1, en tanto no existan datos adicionales para calibrarlas. Las extrapolaciones deben tomarse con las reservas del caso.

Los resultados reportados en las figuras 2 a 9, relativamente altos para las condiciones del bosque de interés, e.g., existencias de $700 \mathrm{~m}^{3} /$ ha e incrementos instantáneos de aproximadamente 25-30 $\mathrm{m}^{3} /$ ha/año (con máximo alrededor de 15 años de edad) y promedio anuales de 15-20 $\mathrm{m}^{3} / \mathrm{ha}$ /año (con máximo alrededor de los 29 años), deben tomarse con prudencia y tener presente que corresponden a estimaciones procuradas por un conjunto de ecuaciones obtenidas a partir de cierta geometría de datos usados en su construcción, correspondientes a una muestra parcial de las condiciones ambientales y silvícolas del lugar.

\footnotetext{
$4 \quad$ Zepeda et al. (1994) y Zepeda y Domínguez (1994), usaron satisfactoriamente 4.5 (90 m²/ha).

5 Cabe anotar que Arteaga (1985) reporta áreas basales para Pinus patula de Zacatlán, Puebla, de poco más de $100 \mathrm{~m}^{2} / \mathrm{ha}$, no confirmadas por otros autores. Adicionalmente, no se encontraron reportes similares en la literatura disponible para Pinus montezumae.
} 


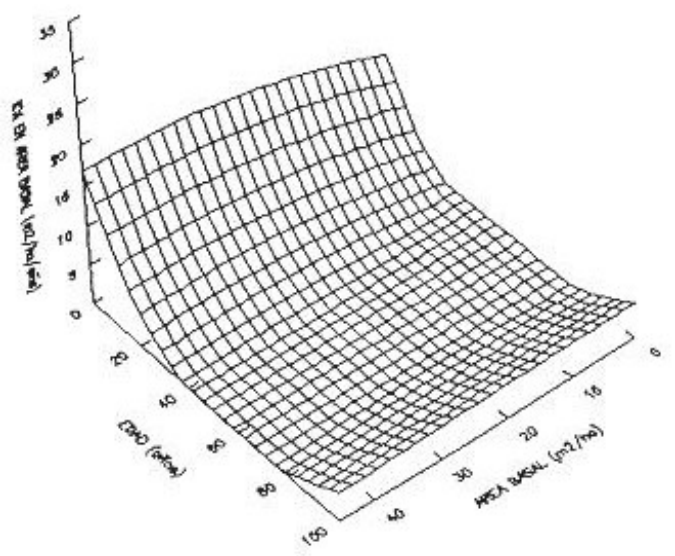

Figura 2. Comportamiento del incremento instantáneo en área basal para Pinus montezumae Lamb. de San Juan Tetla, Puebla.

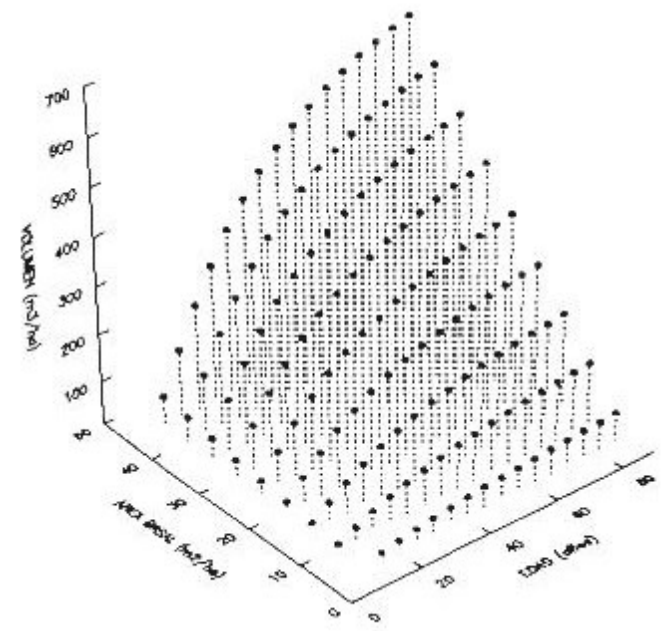

Figura 3. Comportamiento del rendimiento volumétrico de Pinus montezumae Lamb. de San Juan Tetla, Puebla.

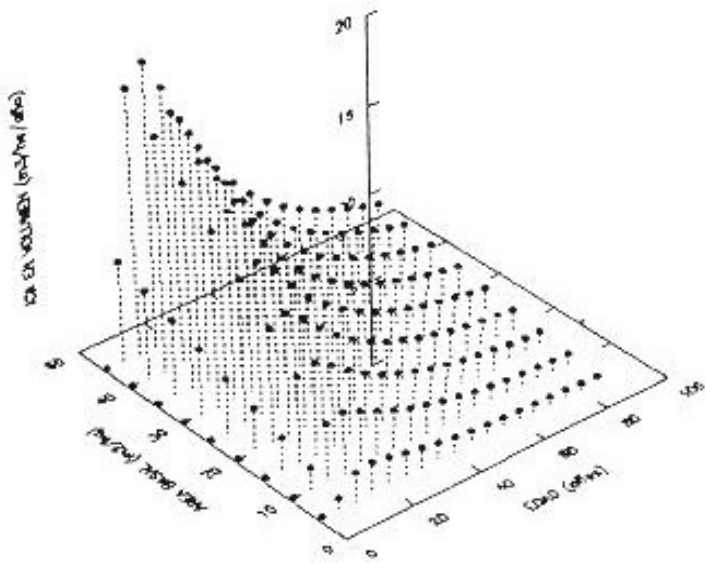

Figura 4. Comportamiento del incremento instantáneo en volumen de Pinus montezumae Lamb. de San Juan Tetla, Puebla.

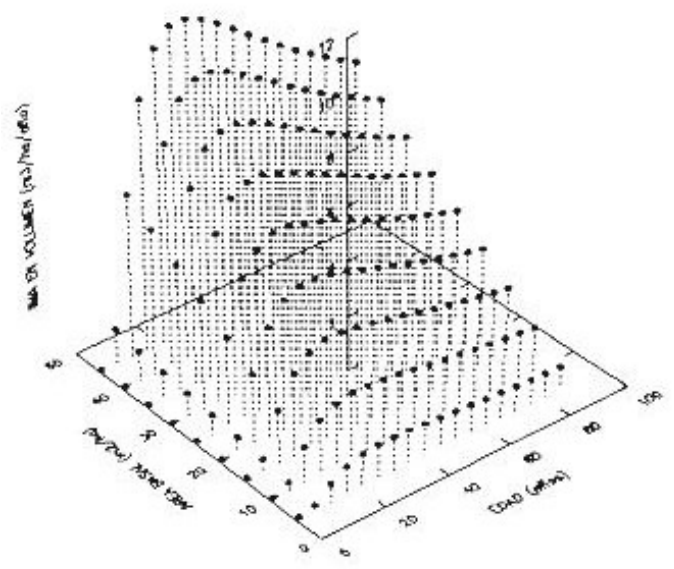

Figura 5. Comportamiento del incremento medio anual en volumen de Pinus montezumae Lamb. de San Juan Tetla, Puebla. 


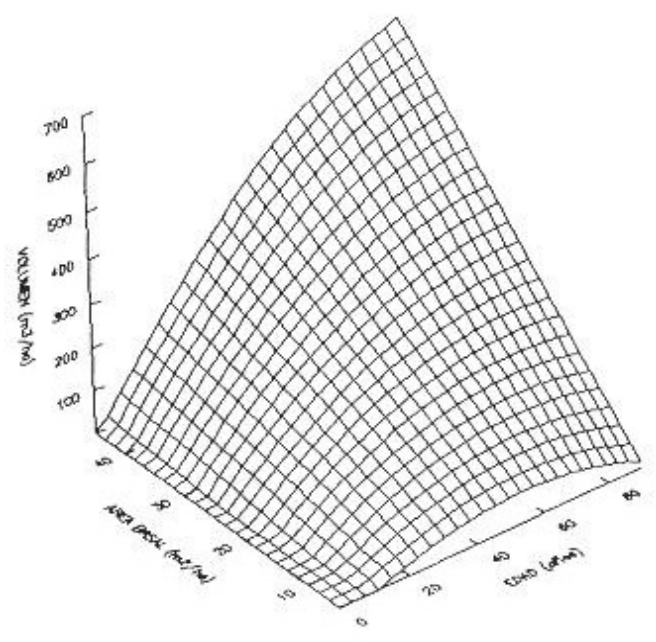

Figura 6. Relación entre el volumen total, el área basal y la edad en Pinus montezumae Lamb. de San Juan Tetla, Puebla. IS $=25 \mathrm{~m}$.

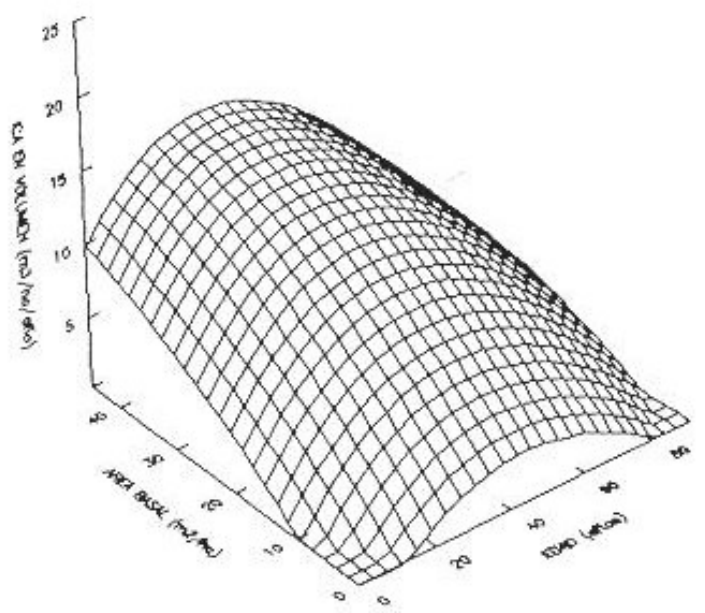

Figura 7. Relación entre el incremento anual en volumen, el área basal y la edad en Pinus montezumae Lamb. de San Juan Tetla, Puebla. IS $=25 \mathrm{~m}$.

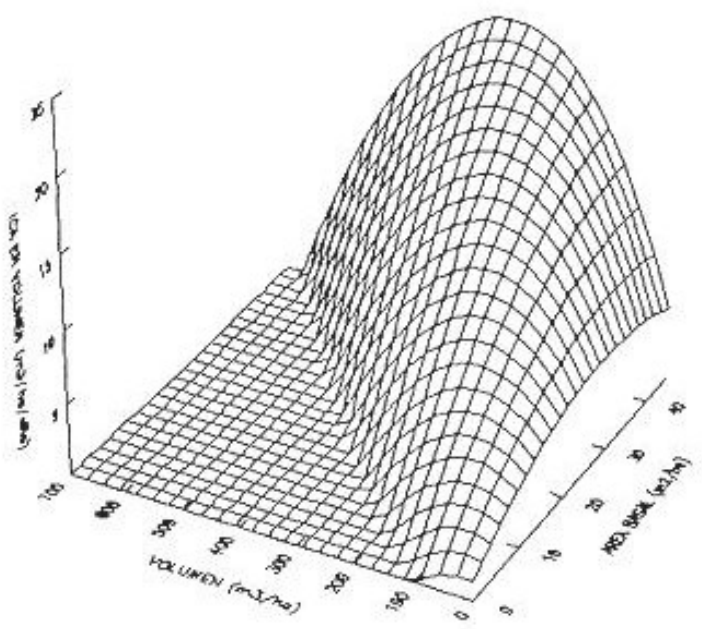

Figura 8. Relación entre el incremento anual en volumen, el volumen por hectárea y el área basal en Pinus montezumae Lamb. de San Juan Tetla, Puebla. IS = $25 \mathrm{~m}$.

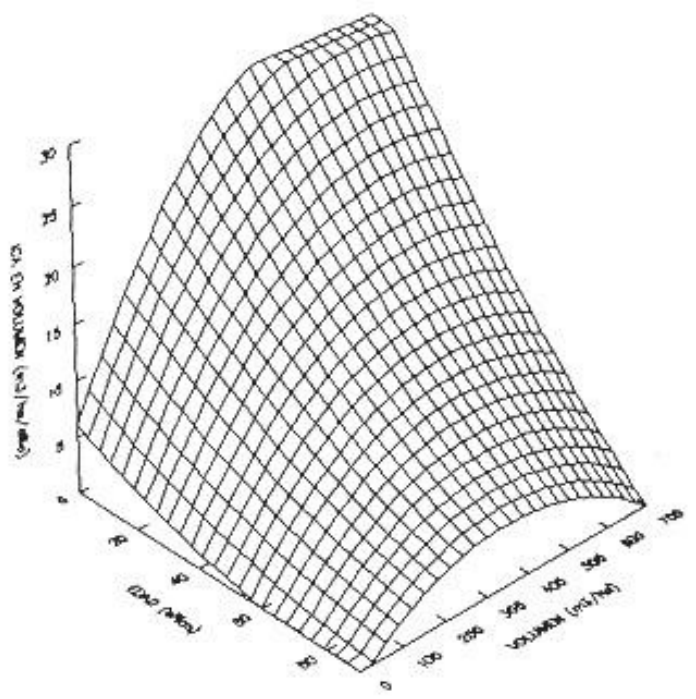

Figura 9. Relación entre el incremento anual en volumen, la edad y el volumen por hectárea en Pinus montezumae Lamb. de San Juan Tetla, Puebla. IS = $25 \mathrm{~m}$. 


\section{CONCLUSIONES Y SUGERENCIAS}

Tomando en cuenta las limitaciones posibles de las ecuaciones que sirvieron de base para el presente análisis de rendimientos maderables, se arribó a las siguientes consideraciones:

C Se confirma que el crecimiento y el rendimiento maderables deben ser modelados matemáticamente atendiendo a la compatibilidad que determina la interdependencia biológica existente entre ellos, con la mayor precisión posible, por lo que debe existir compatibilidad analítica manifiesta entre las funciones preparadas para estimar el incremento y rendimiento maderables, como en este caso.

C Los resultados aquí obtenidos pueden apoyar a la planeación, en forma cauta, del manejo forestal de poblaciones forestales similares a las empleadas para construir las ecuaciones, pues permiten evaluar, aún de manera inicial, algunas relaciones existentes entre la densidad, la productividad y la producción, válidas y aplicables únicamente a la especie y condición ambiental de interés (Figs. 2-9); mismas que no deben tomarse en forma dogmática, ni generalizarse.

C Específicamente pueden ser usados, sin exagerar, para estimar el monto del rendimiento maderable, para niveles variables de densidad residual y para las calidades de sitio de interés; así como auxiliar en la estimación de la producción maderable, en la definición de longitudes de turno -físicos y financieros-, en la valoración inicial de rodales, en el diseño de regímenes silvícolas y, entre otros, en el control del rendimiento de madera; en todos los casos, únicamente para condiciones biofísicas similares a las del Plan de Marines, Chiautzingo, Puebla.

C Con todo y las limitaciones del anamorfismo patente en algunas de las relaciones dasométricas presentadas, el enfoque metodológico adoptado y la estructura de modelo propuesta, permiten generar estimaciones eficientes del incremento y rendimiento maderable, y de los efectos sobre ellos de la densidad y la calidad de sitio de los rodales, lo que sugiere su adopción para estudiar tales relaciones en otras especies y regiones forestales importantes de México.

C Se considera que para superar la etapa del manejo forestal "descriptivo", es necesario mejorar los métodos de estimación de rendimientos maderables, a base del criterio profesional, en donde se aplica al bosque "lo que el bosque pide", basados algunos de ellos en métodos de estimación injustificables, por métodos cuantitativos más racionales, empleados cotidianamente en países de silvicultura más avanzada que la nuestra y que no resultan más onerosos o difíciles que los otros, sólo que hay necesidad de aprenderlas

\section{REFERENCIAS}

Acosta M., M. 1991. Modelo de crecimiento para Pinus montezumae Lamb., en el CEF San Juan Tetla, Puebla. Univ. Autónoma Chapingo. Div. Ciencias Forestales. Programa de Postgrado. Tesis de Maestría en Ciencias. Chapingo, México. 88 p.

Alder, D. 1979. A distance-independient tree model for exotic conifer plantations in East Africa. For. Sci. 25(1): 59-71.

Arteaga M., B. 1985. Indice de sitio para Pinus patula Schl et. Cham., en la 
región Chignahuapan-Zacatlán, Puebla. Tesis de Maestría en Ciencias. Colegio de Posgraduados. Montecillo, México. 181 p.

Clutter, J.L. 1963. Compatible growth and yield models for loblolly pine. For. Sci. 9(3): 354-371.

Clutter, J.L., J.C. Forston, L.V. Pienaar, G.H. Brister y R.I. Bailey. 1983. Timber management; A quantitative approach. Wiley, NuevaYork. 333 p.

Zepeda B., E.M., A. Domínguez P. y O. Estrada M. 1994. Ecuaciones para estimar rendimientos maderables de Pinus arizonica Engl., en el noroeste de Chihuahua. In: Resúmenes. Simposio. y II Reunión Nacional sobre
Silvicultura y Manejo de Recursos Forestales: Retos y perspectivas. Montecillo, Edo. de México, 6-9 sept. 1994. p: 44.

Zepeda B., E.M. y A. Domínguez P. 1994. Rendimientos maderables de Pinus arizonica Engl., en El Poleo, Chihuahua. In: Resúmenes. Simposio. y II Reunión Nacional sobre Silvicultura y Manejo. de Recursos Forestales: Retos y perspectivas. Montecillo, México, 6-9 sept. 1994. p: 45.

Zepeda B., E.M. y A. Domínguez P. 1998. Niveles de incremento y rendimiento maderable de poblaciones naturales de Pinus arizonica Engl., de El Poleo, Chihuahua. Madera y Bosques, 4(1): 27-39. 